\title{
Weak Dominance Drawings for Directed Acyclic Graphs
}

\author{
Evgenios M. Kornaropoulos ${ }^{1,2}$ and Ioannis G. Tollis ${ }^{1,2}$
}

1 Department of Computer Science, University of Crete, Heraklion, Crete, Greece

2 Institute of Computer Science, Foundation for Research and Technology-Hellas

\{kornarop, tollis\}@ics.forth.gr

The dominance drawing method has many important aesthetic properties, including small number of bends, good vertex placement, and symmetry display [1. Furthermore, it encapsulates the aspect of characterizing the transitive closure of the digraph by means of a geometric dominance relation among the vertices. A dominance drawing $\Gamma$ of a planar st-graph $G$ is a drawing, such that for any two vertices $u$ and $v$ there is a directed path from $u$ to $v$ in $G$ if and only if $X(u) \leq X(v)$ and $Y(u) \leq Y(v)$ in $\Gamma[1$. Here we study weak dominance drawings where for any two vertices $u$ and $v$ if there is a directed path from $u$ to $v$ in $G$ then $X(u) \leq X(v)$ and $Y(u) \leq Y(v)$ in $\Gamma$.

The notion of dominance drawing dimension of a directed acyclic graph $G$ (denoted as $\operatorname{dim}(G)$ ) is defined as the value of the smallest $k$ for which a $k$ dimensional dominance drawing of $G$ can be obtained 2. If a graph $G$ has $\operatorname{dim}(\mathrm{G})>2$ then there is at least one pair of vertices $u, v \in V$ such that $X(u) \leq$ $X(v)$ and $Y(u) \leq Y(v)$ in $\Gamma$, while neither $u$ can reach $v$, nor $v$ can reach $u$. In this case we say that there is a falsely implied path (or fip) from $u$ to $v$. Let $\operatorname{inc}(G)$ be the number of pairs of vertices $u, v$ such that neither $u$ is reachable from $v$, nor $v$ is reachable from $u$. The following two upper bounds hold: (a) $\min _{\Gamma} \operatorname{fip}(\Gamma) \leq \operatorname{inc}(G)-\left\lceil\frac{2 \operatorname{inc}(G)}{\operatorname{dim}(G)}\right\rceil,(\mathrm{b}) \min _{\Gamma} \operatorname{fip}(\Gamma) \leq \operatorname{inc}(G)-(\operatorname{dim}(G)-2)$. The challenge of this problem is to minimize the number of fips. The existence of falsely implied paths is the trade off in drawing graphs with $\operatorname{dim}(G)>2$ in the two dimensional plane using the concept of dominance. The intersection of two topological sortings $t_{1}, t_{2}$ of vertices in $V$ is the set $I=\left\{(u, v) \mid t_{1}(u)<\right.$ $\left.t_{1}(v), t_{2}(u)<t_{2}(v)\right\}$. The corresponding decision problem is defined as follows:

\section{(WDD) WEAK DOMINANCE DRAWING FOR DAGs}

INSTANCE: A directed acyclic graph $G=(V, E)$ and a positive integer $C$ such that $|E| \leq C \leq \frac{|V|(|V|-1)}{2}$.

QUESTION: Does there exist a collection of two topological sortings $t_{1}, t_{2}$ of $V$ such that their intersection has cardinality $C$ or less?

The NP-complete problem LINEAR EXTENSION DIAMETER reduces to the WEAK DOMINANCE DRAWING FOR DAGs problem. For a more detailed description on the above see [3. Hence, we propose three heuristic algorithms that seem to perform well in practice. In the following algorithms the topological sorting $t_{1}$ is given as an input and it remains fixed. Therefore, the goal is to construct a topological sorting $t_{2}$ such that the quantity fip $\left(t_{1}, t_{2}\right)$ is minimized. 

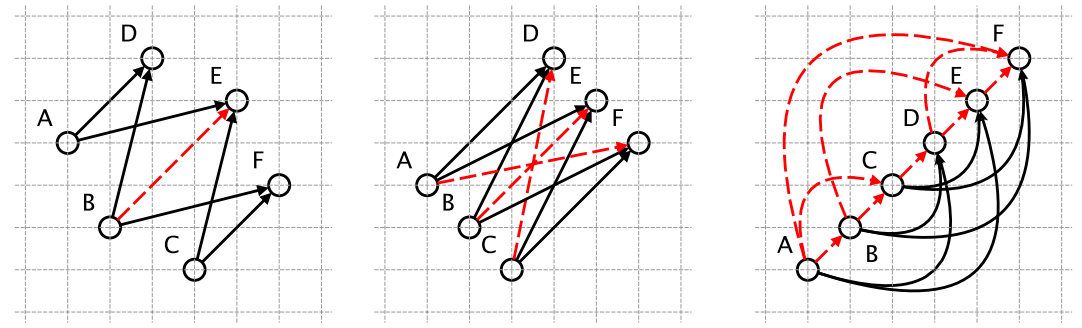

Fig. 1. Three different weak dominance placements of crown graph $C_{3}$. Falsely implied paths appear in red with broken line. In the left figure we have one fip, in the middle we have three fips, while in the right we have nine fips.

The first algorithm is based on paths that cover $G$. The second is a local search algorithm where we choose $t_{2}$ to be the same as $t_{1}$ for initialization. As a next step, the algorithm tests if a local swap between two incomparable and consecutive vertices in $t_{2}$ results in a decrease of the number of fips. The process is repeated until we reach a locally optimal solution. Since the swap is performed only if the number of fips strictly decreases, the algorithm reaches a locally optimal solution in at most $\left(\begin{array}{l}n \\ 2\end{array}\right)$ improvement steps.

The third algorithm, named Max-Rank, follows a greedy approach. In the algorithm Max-Rank the following procedure is repeated until every vertex of the dag is in the topological sorting $t_{2}$ : choose a source $u$ with the highest position in the fixed $t_{1}$ (also called rank), place $u$ in the under-construction topological sorting, remove $u$ from the graph and update the set of sources. It is greedy in the following sense: among all the potential available sources in a given iteration, the choice of $u$ avoids all the fips between $u$ and the available sources. With the use of a maxheap the algorithm Max-Rank runs in $O(n \log n+m)$.

Surprisingly the Max-Rank algorithm constructs a topological sorting according to the embedding used by the Straight-Line-Draw algorithm for planar stgraphs [1], as described below.

Theorem 1. Let $G$ be a directed acyclic planar st-graph. Let $X, Y$ be the topological sortings constructed by procedures LabelX and LabelY, respectively, of the algorithm Straight-Line-Draw [1. The output $t_{2}$ of $\operatorname{Max}-\operatorname{Rank}(G, X)$ (resp. $\operatorname{Max}-\operatorname{Rank}(G, Y))$ is identical to $Y$ (resp. $X)$.

\section{References}

1. Di Battista, G., Tamassia, R., Tollis, I.G.: Area Requirement and Symmetry Display of Planar Upward Drawings. Discrete and Comput. Geom. 7(4), 381-401 (1992)

2. Eades, P., ElGindy, H., Houle, M., Lenhart, B., Miller, M., Rappaport, D., Whitesides, S.: Dominance Drawings of Bipartite Graphs (1993) (manuscript)

3. Kornaropoulos, E.M., Tollis, I.G.: Weak Dominance Drawings and Linear Extension Diameter, arXiv:1108.1439 (2011) 